\title{
Oral Health Approach to Patients with Dementia
}

\section{Joana Braamcamp* and Ricardo Faria-Almeida}

Faculty of Dentistry, University of Porto, Portugal

*Corresponding Author: Joana Braamcamp, Faculty of Dentistry, University of Porto, Portugal.
Received: July 19, 2021

Published: August 21, 2021

(C) All rights are reserved by Joana

Braamcamp and Ricardo Faria-Almeida.

\section{Abstract}

Dementia is a severe public health issue that affects millions of people in the world. The large number of individuals diagnosed with dementia results in the common presence of these patients in a dental medicine environment.

The extensive study of bibliographic material obtained from a search with the terms "Alzheimer's disease", "dementia", "communication", "gerodontology", "geriatric dentistry", "dementia-friendly", "dental care" and "cognitive impairment" allowed to gather the latest information on the topic.

There is an urgent need to develop functional performance algorithms that are validated and tested in the treatment of dementia patients at the dental clinic.

Keywords: Alzheimer's Disease; Dementia; Communication; Gerodontology; Geriatric Dentistry; Dental Care; Cognitive Impairment; Dementia-Friendly

\section{Introduction}

The term "dementia" refers to a set of disorders associated with brain injuries, often degenerative, mainly defined by the presence of cognitive, psychological and behavioral dysfunctions of such severity that they affect autonomy and the ability to carry out daily activities, such as personal hygiene and oral health care. The compromised oral hygiene in this population is proven by different epidemiological studies, which reveal low rates of oral health care among patients with dementia [1,2].

The increasing prevalence of dementia in society results in a frequent contact with these patients in a dental medicine context and might pose a real challenge to dentists. The treatment of pa- tients with dementia requires an adaptation of the procedures and clinical approaches to the existing needs. Adapting a clinical approach to a patient with dementia means that the dentist should be able to communicate with the patient and with his/her legal representative and conceive a treatment plan that is adequate to the oral needs of the patient and to the progression of the diagnosed dementia syndrome. Such treatment plan should include preventive oral hygiene measures to be adopted by the patient, by his legal representative or by the health professionals, aimed at ensuring the quality of life and the comfort of the patient with dementia [3-8].

\section{Materials and Methods}

The bibliographic material selected for this study was taken from the PubMed platform. A research with the terms "Alzheimer's 
disease", "dementia", "communication", "gerodontology", "geriatric dentistry", "dementia-friendly", "dental care" and "cognitive impairment" allowed to gather scientific papers about the topic. In a preliminary stage, the papers were selected based on their title and abstract, and then by reading them in full. After choosing the papers, the information was organized in categories and put into context to ensure a clear and simplified approach to the topic.

\section{Alzheimer's disease}

Alzheimer's is the most common form of dementia, and it affects 60 to $80 \%$ of patients with dementia syndrome. It is a neurodegenerative disorder, and the signs and symptoms of its progression are memory loss, decreased attention and sense of orientation, reduced communication skills and visual acuity, apraxia, struggle to carry out tasks, behavioral changes, difficulty with social interactions and an altered sensory perception $[1,3,7]$.

The progression of Alzheimer's has a negative effect on oral health due to the limitations in the act of oral hygiene, namely: xerostomia inked to the use of medications, limitations associated with the difficulty in adhering to dental treatments and in seeking medical help. Some authors describe this group of patients as having high rates of bacteria plaque, periodontitis, stomatitis, root caries, mucositis and candidiasis. These conditions can cause pain or discomfort to the patient, interfering with his quality of life. Paying attention to the management of conditions that might cause pain to the patient is especially needed in the advanced stages of a neurodegenerative disease, since the patient might not be able to transmit these symptoms to the health professionals. The possible signs of oral pain in patients with dementia are listed in table 1 (Table 1) [7]. It is also important to refer that, despite the poor oral health rates among this group, some epidemiological studies suggest that the percentage of patients that seeks medical help is significantly low [7-11].

The deterioration of oral health in people with dementia has consequences with regard to the health condition and well-being of these patients. A poor oral health might have systemic consequences or even accelerate the neurodegenerative process associated with Alzheimer's, as some studies have tried to demonstrate. Some of the consequences for the systemic health are diabetes and heart diseases, whose association with periodontitis is accepted among the scientific community. The accumulation of bacterial plaque has

\begin{tabular}{|l|}
\hline Factors indicative of oral pain in patients with dementia \\
\hline Refusal to eat (particularly hard or cold foods) \\
\hline Constant pulling at the face \\
\hline Increased drooling \\
\hline Leaving previously worn dentures out of the mouth \\
\hline Increased restlessness \\
\hline Moaning or shouting \\
\hline Disturbed sleep \\
\hline Refusal to co-operate with normal daily activities \\
\hline Self-injurious behaviour \\
\hline Aggressive behaviour towards carers \\
\hline
\end{tabular}

Table 1: Possible signs of the presence of oral pain in patients with dementia according to Marchini 2019 [7].

also been associated with the possibility of developing pneumonia by aspiration, which is one of the main causes of death in advanced stages of dementia [9-17].

The complications associated with the deterioration of oral health might be prevented with an appropriate medical follow-up. Prevention is possible, but it requires raising awareness among health professionals to the need of referring patients to odontology services, and also the need for training dentists and for the validation of clinical approaches to be adopted specifically with these patients.

\section{Communicating with dementia patients}

The way how patients with dementia typically behave during a visit to the doctor turns the diagnosis and treatment into a challenge for the dentist. The agitation, anxiety, confusion and aggressiveness are common indicators of a patient with dementia in a dental examination. The expression of these signs varies according to the stage of the neurological disease, the patient's past experiences at a dental clinic and the presence of the legal representative in the room [18-22].

The neurologic diagnosis of dementia must be followed by a referral of the patient to the right experts, in order to assure a multidisciplinary approach. One of these experts is the dentist, whose diagnosis and treatment plan must be made in the early stages of the disease. Regular routine check-ups are fundamental, as they 
turn the medical environment into a familiar place and allow a proper monitoring of the patient's oral state. Cerajewska and her colleagues reinforce the benefits of the presence of the legal representative at dental appointments [3-8].

An effective communication with the patient is a key factor to achieve an adequate dental treatment. Since dementia might affect the ability of these individuals to express themselves, an unfulfilled attempt at communication might cause frustration and irritation in the patient. As such, it is suggested an adaptation of the algorithms used in Nursing to the dental clinic in order to seek a "dementiafriendly" treatment. The VERA algorithm (validation, emotion, reassure, activity) was developed for nurses in a way to establish empathy and communication with Alzheimer's patients. This algorithm consists in a simple guide that allows the health professional to answer in a sympathetic way to a confuse patient and try to understand the motivations behind the witnessed behavior, helping the patient to manage them. The validation, emotion, reassurance and activity are elements that have proven to be functional in the management of dementia patients; however, their applicability in dental medicine must be tested. The stages of action of the algorithm are described in table 2 (Table 2) [23-25].

Developing empathy and complicity with the patient is important and might facilitate the collaboration and acceptance of medical procedures, reducing any anxiety or confusion that the patient might be manifesting. In this way, the dentist should be able to render all the information as clearly and simple as possible, slowly and in a tone of voice that allows the patient to hear and understand what is said. During the appointment, the dentist should explain each procedure calmly, in order to tranquilize the patient. The appointments must be as brief as possible, and he must strive to transmit confidence and tranquility to the patient. Knowing his interests and tastes might be useful to the emotional management during the appointment $[18,27]$.

Nevertheless, with the progression of the disease, it is natural that the patient's cooperation decreases, and there might be a necessity to resort to methods of sedation at the clinic or general anesthesia at the hospital in order to enable the execution of the necessary dental procedures $[3,18]$.

\section{Elaborating a dental treatment plan}

A dental treatment plan for a patient with Alzheimer's must be realistic and take into consideration the progression of the de-

\section{VERA Algorithm}

The validation stage refers to valuing the behavior of patients with dementia. in this way, instead of seeing a behavior as a sign of a degenerative

Validation disease, the reasons that lead the patient to adopt a certain attitude must be considered. one should not ignore, then, actions without apparent meaning, but consider reasons inherent to them

The second phase of the algorithm consists of validating the experience, trying to meet the patient's emotional aspect. the professional must be aware of the patient's body language, gestures and facial expressions that allow him to understand what information the patient is trying to convey. likewise, the health professional must also be aware of how to respond to such information. the patient may not be able to perceive the content verbalized by the professional, making the body language and way of presenting the verbal content have an added relevance in this type of communication

In the phase of reassurance, the objective is to transmit tranquility and security to the patient, seeking to establish a bond of trust with the

Reassure health professional. it can be achieved with the words "it will be all right" or with a smile or a kind gesture

The activity stage reinforces the importance of involving the patient in a structured activity, giv-

Activity ing him a sense of occupation. the activity could consist of a moment of reflection while you are sitting holding the health professional's hand

Table 2: VERA Algorithm - description of the stages of action [23].

generative disease. The key to success is "high quality, low maintenance", as suggested by Cerajewska and her colleagues in their article published in 2019 [4].

The treatment plan should enable treatment and prevention, ensuring the patient's quality of life during the progression of dementia. Despite the lack of validated performance protocols, there are some publications that suggest oral treatment plans for these patients. Dougall and his collaborators [11] presented the following treatment guidelines:

1) Start preventive measures as soon as possible;

2) Perform all necessary dental treatments; 
3) Professional cleaning of removable or fixed dentures;

4) Control visits every 3 months or 6 months, as needed by the patient;

5) Emotional management of the patient during oral treatments;

6) Educate the legal guardian about the patient's oral hygiene measures.

The Alzheimer's disease has been associated with periodontal disease, thus one of the main concerns of the dentist must be the periodontal diagnosis and treatment, whenever justifiable. Periodontal health must be assessed at each routine check-up, in a way to ensure control over the accumulation of bacterial plaque and tartar [18].

When creating a treatment plan, the dentist should bear in mind the prognosis and the duration of the dental procedures, avoiding the conduction of highly demanding dental treatments at a later stage of Alzheimer's. In this regard, we must consider the results of a study conducted by Caplan and his collaborators [28], which showed that dental restorations with composite resins in elder patients last, on average, 6.2 years. The approach to extensive injuries caused by caries or root caries must be thought over with care, since teeth with extensive restorations and a bad prognosis might cause problems in more advanced stages and increase morbidity in patients. There are no defined protocols as to the approach that must be adopted; therefore the decision on whether to treat or extract the tooth falls on the dentist, who should always bear in mind the progression of dementia $[4,28]$.

The oral rehabilitation of totally or partially edentulous patients is a hot topic and must be thought over for each patient individually. A study conducted by Packer and his team [29] showed that a fixed rehabilitation with dental implants improved the quality of life of Parkinson patients. However, according to Cerajewska and her colleagues $[3,4]$, the surgery to place dental implants isn't usually recommended for patients with Alzheimer's; again, one must consider the current stage of the neurodegenerative disease in each patient while deciding on an oral rehabilitation procedure.

The treatment plan must be consistent with the stage of Alzheimer's and with the patient's willingness to cooperate with regard to the dental procedures. In this regard, Marchini and his colleagues [7] published recommendations of dental treatments to each stage of Alzheimer's (Table 3). These recommendations must still be tested and validated; thus, while no planning protocols are implemented according to each stage of the disease, the dentist must make his judgment based on the patient's history and, in the best way he can, ensure the patient's present and future quality of life. The high prevalence rates associated with this neurologic disease results in an urgent need to define functional performance protocols to each stage of Alzheimer's, validating them in a dental medicine context.

\begin{tabular}{|c|c|}
\hline $\begin{array}{c}\text { Alzheimer's } \\
\text { disease } \\
\text { stages }\end{array}$ & Recommended dental care \\
\hline Mild & $\begin{array}{l}\text { Early intervention; } \\
\text { Comprehensive examination, including radio- } \\
\text { graphs, formulation of a dental treatment plan, } \\
\text { and an oral hygiene maintenance plan; } \\
\text { Treatment is similar to regular treatment, can } \\
\text { usually be done in dental office; } \\
\text { Treatment is time limited and urgent as the } \\
\text { patient's ability to cooperate will deteriorate. }\end{array}$ \\
\hline Moderate & $\begin{array}{l}\text { The focus should be on maintenance (if early } \\
\text { intervention was provided); } \\
\text { Treatment may be done in the dental office, } \\
\text { with or without oral sedation; } \\
\text { At risk teeth should be extracted or crowned at } \\
\text { this stage. }\end{array}$ \\
\hline Severe & $\begin{array}{l}\text { The focus should be on maintenance (if early } \\
\text { intervention was provided); } \\
\text { Treatment needs to be provided at patient } \\
\text { home/institution, or under deep sedation or } \\
\text { general anesthesia. }\end{array}$ \\
\hline
\end{tabular}

Table 3: Dental treatment recommendations according to stage of Alzheimer's disease [7].

Auxiliary measures of oral hygiene to the patient with dementia

The success of the dental treatment depends on the patient's oral hygiene habits and on primary care. Thus, considering the already mentioned limitations and characteristics of dementia patients, it is suggested that the dentist provides auxiliary measures of oral hygiene to the patient's legal representative [30]. 
Auxiliary measures of oral hygiene are preventive measures that help patients to preserve their oral health at home. The benefits of using electric toothbrushes, 5000 ppm fluoride SLS-free toothpaste and $0.2 \%$ chlorhexidine mouthwashes have already been mentioned by some authors. The use of sticky notes to remind the patient about his daily oral hygiene might be suggested, and he should also be reminded several times about any appointments at the dental clinic $[4,18,31]$.

The progression of degenerative diseases causes patients, mainly those who are at later stages of the disease, to be dependent on their legal representative or on health professionals at home. With this in mind, some authors refer the importance of teaching legal representatives how to perform oral hygiene and answer any questions that might arise with regard to oral health care. When patients are hospitalized or at nursing homes, their oral hygiene is the responsibility of health professionals, so these must be aware of the importance of preserving oral health and receive adequate training for that effect $[8,32,33]$.

\section{Conclusion}

The increasing number of people with dementia in our society calls for an adaptation of dental medicine procedures to this $21^{\text {st }}$ century challenge. The high prevalence rates of this neurologic condition suggest that only one part of these patients is having an appropriate medical follow-up. As a result, there is an urgent need to create, validate and test functional performance algorithms to treat patients with dementia at the dental clinic.

\section{Bibliography}

1. Gale AS., et al. "Dementia". The American Journal of Medicine 131.10 (2018): 1161-1169.

2. Aragón F., et al. "Oral health in Alzheimer's disease: a multicenter case-control study". Clinical Oral Investigation 22 (2018): 3061-3070.

3. Cerajewska TL., et al. "Dementia friendly dentistry for the periodontal patient. Part 1: recognizing and assessing patients with dementia”. British Dental Journal 227 (2019): 563-569.

4. Cerajewska TL., et al. "Dementia friendly dentistry for the periodontal patient. Part 2: ethical treatment planning and management". British Dental Journal 227 (2019): 570-576.
5. Geddis-Regan A., et al. "The impact of dementia on oral health and dental care, part 2: approaching and planning treatment". Primary Dental Journal 9.2 (2020): 31-37.

6. Hamza SA., et al. "Oral health of individuals with dementia and Alzheimer's disease: A review". Journal of Indian Society of Periodontology 25.2 (2021): 96-101.

7. Marchini L., et al. "Oral health care for patients with Alzheimer's disease: an update". Special Care Dentisry Journal (2019): 1-12.

8. Rozas NS., et al. "Strategies to improve dental health in elderly patients with cognitive impairment". The Journal of the American Dental Association 148.4 (2017): 236-245.

9. Delwel S., et al. "Oral hygiene and oral health in older people with dementia: a comprehensive review with focus on oral soft tissues". Clinical Oral Investigations 22 (2017): 93-108.

10. Ferreira MC., et al. "Impact of periodontal disease on quality of life: a systematic review”. Journal of Periodontal Research 52.4 (2017): 651-665.

11. Dougall A and Fiske J. "Access to special care dentistry, part. 9. Special care dentistry services for older people". British Dental Journal 205.8 (2008): 421-434.

12. Lin JW., et al. "Examining the association between oral health status and dementia: A nationwide nested case-controlled study". Experimental Biology and Medicine 245.3 (2020): 231244.

13. Alvarenga MOP., et al. "Is There Any Association Between Neurodegenerative Diseases and Periodontitis? A Systematic Review". Frontiers in Aging Neuroscience 13 (2021): 1-13.

14. Diaz-Zuñiga J., et al. "Serotype b of Aggregatibacter actinomycetemcomitans triggers pro-inflammatory responses and amyloid beta secretion in hippocampal cells: a novel link between periodontitis and Alzheimer's disease?" Journal of Oral Microbiology 11.1 (2019): 1-11.

15. Dioguardi M., et al. "The Role of Periodontitis and Periodontal Bacteria in the Onset and Progression of Alzheimer's Disease: A Systematic Review". Journal of Clinical Medicine 9.495 (2020): 1-21. 
16. Kamer AR., et al. "Periodontal disease as a possible cause for Alzheimer's disease". Periodontology 200083 (2019): 242271.

17. Rong X., et al. "Chronic Periodontitis and Alzheimer Disease: A Putative Link of Serum Proteins Identification by 2D-DIGE Proteomics". Frontiers in Aging Neuroscience 12 (2020): 1-10.

18. Edwards JA., et al. "Dementia and dentistry". Special Care Dentistry 42 (2015): 464-472.

19. Kazui H., et al. "Differences of Behavioral and Psychological Symptoms of Dementia in Disease Severity in Four Major Dementias". PLOS One 11.8 (2016): 1-33.

20. Alsaway S., et al. "What is good communication for people living with dementia? A mixed-methods systematic review". International Psychogeriatrics 29.11 (2017): 1785-1800.

21. Bergdahl E., et al. "Depression among the very old with dementia”. International Psychogeriatrics 23.5 (2011): 756-763.

22. Ming Y., et al. "Association of oral health-related quality of life and Alzheimer disease: a systematic review". The Journal of Prosthetic Dentistry 124.2 (2020): 168-175.

23. Blackhall A., et al. "VERA framework: communicating with people who have dementia". Nursing Standard 26.10 (2011): 35-39.

24. Handley M., et al. "Dementia-friendly interventions to improve the care of people living with dementia admitted to hospitals: a realist review". BMJ Open 7 (2017): 1-13.

25. Naughton C., et al. "A feasibility study of dementia communication training based on the VERA framework for pre-registration nurses: Part II impact on student experience". Nurse Education Today 63 (2018): 87-93.

26. Wright CD., et al. "Periodontal Status and Quality of Life: Impact of Fear of Pain and Dental Fear". Pain Research and Management (2017): 1-9.

27. McDermott 0., et al. "The importance of music for people with dementia: the perspectives of people with dementia, family carers, staff and music therapists". Aging and Mental Health 18.6 (2014): 706-716.
28. Caplan DJ., et al. "Dental Restoration Longevity among Geriatric and Special Needs Patients". JDR Clinical and Translational Research 4.1 (2018): 1-8.

29. Packer M., et al. "The potential benefits of dental implants on the oral health quality of life of people with Parkinson's disease". Gerodontology 26 (2009): 11-18.

30. Gao SS., et al. "Oral Health and Care for Elderly People with Alzheimer's Disease". International Journal of Environmental Research and Public Health 17.16 (2020): 1-17.

31. Fjeld KG., et al. "Effect of electric toothbrush on residents' oral hygiene: a randomized clinical trial in nursing homes". European Journal of Oral Sciences 122 (2014): 142-148.

32. Zenthöfer A., et al. "Carers' education improves oral health of older people suffering from dementia - results of an intervention study". Clinical Interventions in Aging 11 (2016): 17551762.

33. Dubar M., et al. "Awareness and practices of general practitioners towards the oral-systemic disease relationship: A regionwide survey in France". Journal of Evaluation in Clinical Practice 26.6 (2019): 1722-1730.

Volume 5 Issue 9 September 2021 (C) All rights are reserved by Joana Braamcamp and Ricardo Faria-Almeida. 